\title{
Importance des interfaces spatiales et temporelles entre les bovins et les glossines dans la transmission de la trypanosomose animale en Afrique de l'O uest
}

\author{
S. de La Rocque ${ }^{1,2} *$ Z. Bengaly ${ }^{1}$ J.F. Michel ${ }^{1,2}$

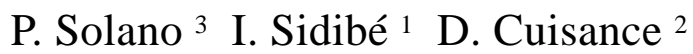

\begin{abstract}
Mots-clés
Bovin - Glossina - Trypanosomose Epidémiologie - Environnement - Agriculture - M éthode d'élevage - Burkina Faso.
\end{abstract}

\begin{abstract}
Résumé
Lors d'une étude globale du risque trypanosomien menée dans la zone agropastorale de Sidéradougou (Burkina Faso), des troupeaux sentinelles issus de deux systèmes d'élevage différents ont été suivis durant deux ans. L'incidence trypanosomienne mensuelle a été mesurée et interprétée en fonction des parcours des troupeaux, de leur pratique d'abreuvement et de leur contact avec les glossines ripicoles (Glossina tachinoides et $\mathrm{G}$. palpalis gambiensis). A Nakaka, village d'éleveurs Peuls, la transmission en saison sèche est assurée aux points d'abreuvement pérennes dans la galerie forestière. En saison des pluies, les glossines ripicoles se dispersent dans les savanes et infectent les animaux jusque dans les villages. A Péfrou, ensemble de campements d'agriculteurs Bobo, les troupeaux sont composés essentiellement de bœufs de traction. Les animaux issus des campements localisés à proximité du cours d'eau s'abreuvent dans les points d'eau des formations riveraines et sont infectés toute l'année. L'incidence est plus élevée en hivernage et en début de saison sèche, période où les glossines sont les plus nombreuses. A l'opposé, les troupeaux des campements éloignés du réseau hydrographique $(3 \mathrm{~km})$ sont abreuvés au puits et ne fréquentent pas les biotopes des glossines. $D$ ans ce paysage essentiellement agricole, les glossines ne se dispersent pas, même en saison humide. L'incidence dans les troupeaux est quasiment nulle. Ces résultats montrent l'importance des interfaces spatiales et temporelles entre les bovins et les glossines dans l'épidémiologie des trypanosomoses en Afrique de l'ouest.
\end{abstract}

\section{INTRODUCTION}

Le fonctionnement du système pathogène incriminé dans la transmission des trypanosomoses animales fait intervenir la classique triade hôte/vecteur/parasite. Au-delà de la répartition des glossines, inféodée à des biotopes particuliers, des études récentes menées dans une zone agropastorale du Burkina Faso ont montré que les glossines ne sont pas partout infectées par les mêmes parasites et que certains sites sont plus dangereux que d'autres. Ces points à risque se situent aux interfaces les plus intenses entre les glossines et les bovins (9).

\footnotetext{
1. Cirdes, BP 454, 01 Bobo-Dioulasso, Burkina Faso

2. Cirad-emvt, Campus de Baillarguet, 34398, Montpellier Cedex 5, France

3. Institut Pierre Richet, BP 1500, Bouaké, Côte d'Ivoire

* Auteur pour la correspondance :

Tél : + 226972287 ; Fax : + 226972320 ; E-mail : stephane.delarocque@cirad.fr
}

Or, la capacité vectorielle telle qu'elle est souvent perçue laisse peu de place à l'hôte vertébré. Il est généralement considéré comme un agent passif, sur lequel les glossines acquièrent les parasites et auquel elles les transmettent. Pourtant, certaines pratiques pastorales permettent de limiter la fréquentation des sites où les glossines abondent ou d'éviter les heures où elles sont actives ; ces pratiques peuvent être interprétées comme des stratégies de défense de l'hôte.

La stratégie du « rendez-vous » (8) entre les acteurs repose sur les interfaces temporelles et spatiales qui se réalisent à la faveur de la croisée des circuits. Les différentes pratiques et les contraintes du milieu favorisent ou empêchent le contact entre les hôtes vertébrés et les vecteurs. La présente étude vise, à travers le suivi de troupeaux « sentinelles », à mieux comprendre l'épidémiologie analytique de parasitoses à transmission vectorielle. Elle décrit la circulation des parasites entre le compartiment vecteur (les glossines) et le compartiment hôte (le bétail) dans des situations contrastées rencontrées dans des savanes soudaniennes d'Afrique de l'Ouest. 


\section{MATERIEL ET METHODES}

\section{Présentation de la zone}

La zone agropastorale de Sidéradougou se situe au sud de BoboDioulasso, au Burkina Faso, à $11^{\circ} 10^{\prime}$ de latitude Nord et $4^{\circ} 19^{\prime}$ de longitude Ouest (figure 1). Elle s'étend sur $3500 \mathrm{~km}^{2}$ au pied de la falaise de Banfora. Elle est représentative de la zone soudanienne, avec des précipitations de l'ordre de $1100 \mathrm{~mm}$ par an.

Elle est constituée de savanes arborées à karité (Butyrospermum paradoxum) et à badamier (Terminalia laxiflora), de formations ripicoles arbustives ou de galeries forestières qui occupent les berges du réseau hydrographique. Dans cette zone, $1200 \mathrm{~km}^{2}$ ont fait l'objet durant les trois dernières années d'un projet de recherche mené par le Cirad et le Cirdes pour identifier et localiser les sites de transmission des trypanosomoses animales (9). Lors de ce travail, un ensemble d'informations relevées sur le terrain ou issues d'analyses de données de télédétection (photographies aériennes, images Spot) a été intégré dans un système d'information géographique $(\mathrm{Sig})$.

\section{Occupation agricole}

L'occupation agricole a été évaluée par l'interprétation d'une image de télédétection à haute résolution (Spot XS, décembre 1996, résolution 20 m x 20 m). Le parcellaire agricole a été cartographié par interprétation visuelle sur l'image satellite brute puis numérisé (figure 1).

\section{Les systèmes d'élevages}

L'effectif bovin de la zone a été recensé et s'élève à 16500 têtes. Différents systèmes d'élevages ont été identifiés $(15,26)$ :

- des éleveurs traditionnels en voie de sédentarisation, d'ethnie Peul, sont propriétaires de troupeaux de grande taille et pratiquent un mode traditionnel d'élevage semi-transhumant ; leurs déplace- ments sont limités et ne concernent souvent qu'une partie du troupeau ; ces éleveurs sont néanmoins grands consommateurs d'espace ; ils vivent dans les zones peu cultivées ;

- des agriculteurs d'ethnies locales anciennement installées (Bobo, Tiefo, Karaboro, Toussian....) possèdent quelques bovins de trait pour une production essentiellement vivrière (maïs, sorgho, petit mil) ;

- des agropasteurs originaires du plateau Mossi au centre du pays, installés depuis une dizaine d'années, ont été attirés par les conditions propices à la culture du coton ; ils acquièrent initialement quelques animaux de trait, capitalisent en quelques années les revenus cotonniers dans des femelles reproductrices et constituent des troupeaux d'une vingtaine d'animaux en moyenne ; ils vivent généralement dans les zones densément cultivées et, lorsque l'espace pastoral est trop réduit, peuvent confier une partie de leur troupeau à des bouviers Peuls.

A chacun de ces systèmes sont associées des stratégies différentes d'utilisation de l'espace, en réponse à la contrainte majeure que représente l'abreuvement des animaux durant la saison sèche (2).

\section{Situation entomologique}

Lors d'enquêtes entomologiques très fines réalisées en 1996 (un piège posé tous les $100 \mathrm{~m}$ le long de $120 \mathrm{~km}$ de réseau hydrographique), deux espèces de glossines ripicoles, G. tachinoides Westwood, 1850 et G. palpalis gambiensis Vanderplanck, 1949 ont été mises en évidence (12). En revanche aucune glossine de savane n'a été capturée lors de cette prospection.

\section{Suivi des troupeaux sentinelles}

Afin d'évaluer le risque de transmission parasitaire auquel sont soumis les troupeaux selon les pratiques pastorales, deux groupes d'animaux sentinelles ont été suivis durant deux ans. Ces troupeaux ont été choisis dans des situations contrastées.

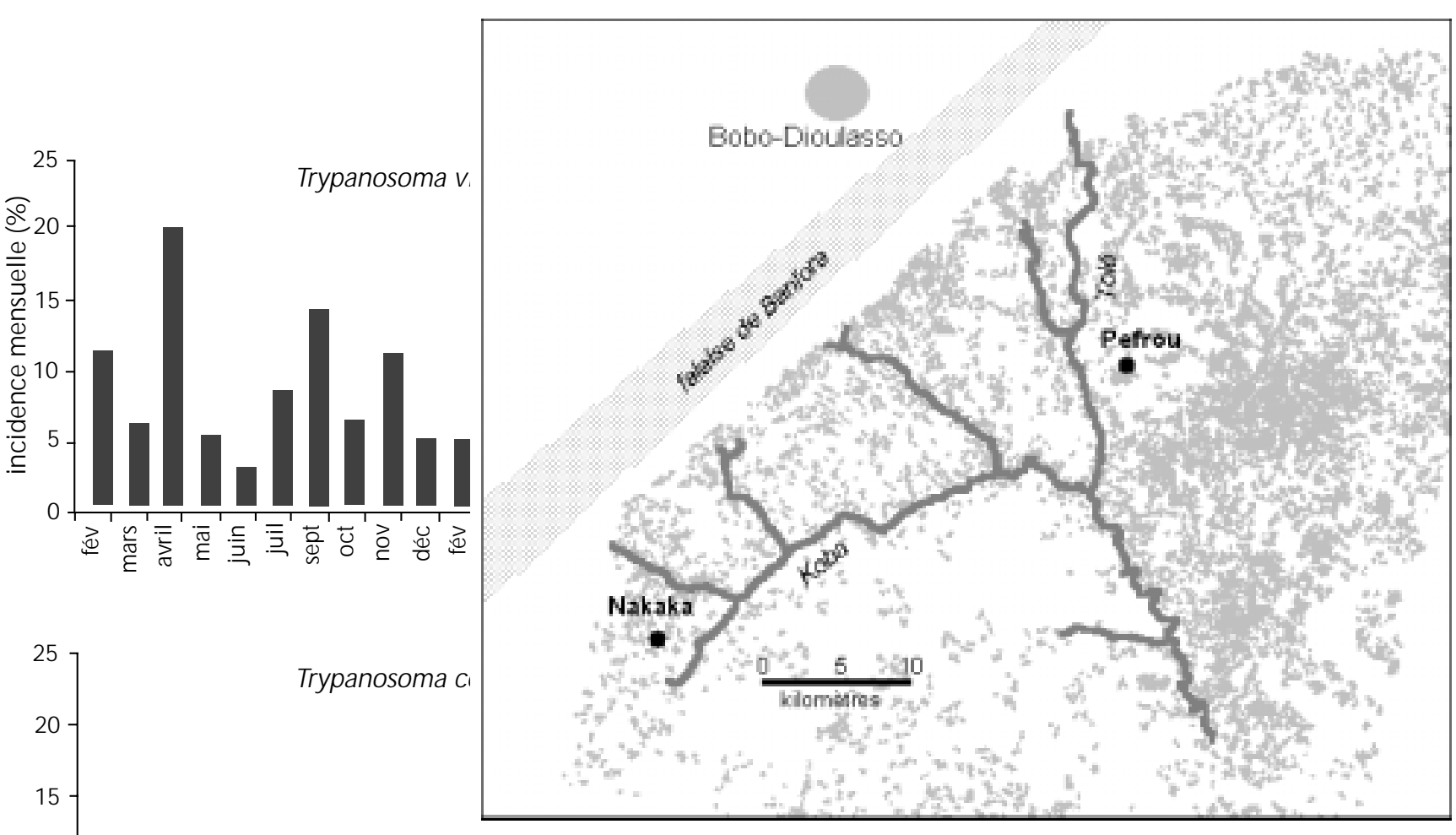

Figure 1 : situation de la zone de Sidéradougou, occupation agricole et localisation des troupeaux sentinelles. 
Le premier troupeau appartient à des éleveurs Peuls qui pratiquent un élevage traditionnel. Leur campement nommé Nakaka se situe dans la partie pastorale au sud-ouest de la zone. Il est composé d'une dizaine de familles sédentarisées depuis plus de vingt ans. Chacune possède entre 15 et 400 animaux, l'effectif bovin total du campement avoisine les 1600 têtes. Les troupeaux de trois familles ont été sélectionnés et les animaux suivis ont été échantillonnés par tirage aléatoire. Au total, 55 animaux ont été retenus et marqués d'une boucle auriculaire.

Le second troupeau est composé d'animaux de plusieurs agriculteurs Bobo localisés sur le terroir du village de Barré, aux abords de la rivière Tolé, dans une zone densément cultivée. Ces agriculteurs possèdent entre 1 et 8 animaux, essentiellement destinés au travail dans les champs. Les animaux de 20 éleveurs ont été retenus, ce qui représente un effectif de 74 têtes.

\section{Protocole de suivi parasitologique}

Chaque troupeau sélectionné a été visité toutes les cinq semaines durant deux ans. Le protocole adopté s'apparente à celui dit de « l'index Bérénil » qui permet de juger de l'incidence trypanosomienne. Lors des visites, une prise de sang à la veine jugulaire a été réalisée sur tous les animaux. Un examen parasitologique a été effectué immédiatement après le prélèvement, en utilisant la technique du buffy coat (centrifugation différentielle en microtubes capillaires, étalement de la phase leucocytaire entre lame et lamelle et examen en microscopie à fond noir) (28). L'observation directe des trypanosomes, leur taille, leur morphologie et leur motilité permettent de réaliser une première diagnose d'espèce, confirmée ultérieurement à l'examen des frottis après coloration (33).

Cette technique est reconnue sensible pour un diagnostic de terrain et permet d'identifier rapidement les animaux dont les parasitémies sont de l'ordre de 300 à 700 trypanosomes par ml $(3,34,35)$. Cependant, les parasitémies peuvent être très faibles et échapper à cet examen direct $(13,24,37)$. Aussi les animaux dont les valeurs de l'hématocrite étaient inférieures à 25 p. 100 ont été considérés comme douteux. Les échantillons positifs et douteux ont été ultérieurement analysés par $\operatorname{Pcr}(32)$.

A l'issue des examens pratiqués sur le terrain, les animaux parasitologiquement positifs ou ayant une valeur de l'hématocrite inférieure à 25 ont été traités à l'acéturate de diminazène (Bérénil ${ }^{\circledR}$ ) à la dose de $7 \mathrm{mg} / \mathrm{kg}$.

\section{Calcul de l'incidence}

Le suivi régulier de troupeaux sentinelles permet d'étudier la vitesse de réinfection par les trypanosomes, donc le niveau d'exposition des animaux à la pression parasitaire. L'acéturate de diminazène est une molécule particulièrement intéressante pour les études d'incidence. Il a un effet curatif immédiat mais son activité trypanopréventive est de courte durée, estimée à une quinzaine de jours. Des visites régulières toutes les cinq semaines laissent à l'animal traité la possibilité de s'infecter dans les vingt jours suivant l'injection (36). A chacune des visites se trouvaient donc, parmi les animaux positifs, des individus qui avaient été positifs et traités à la visite précédente et d'autres qui avaient été négatifs. Le calcul de l'incidence sur trente jours a été réalisé de la manière suivante :

$$
\begin{gathered}
\mathrm{I}=\{[(\mathrm{PN} / \mathrm{N}) \times(30 / \mathrm{d})] \times \mathrm{N} / \mathrm{M}\}+\{[(\mathrm{PT} / \mathrm{T}) \times(30 / \mathrm{d}-15)] \times \mathrm{T} / \mathrm{M}\} \\
\text { ou encore } \mathrm{I}=(30 / \mathrm{N}) \times[(\mathrm{PN} / \mathrm{d})+(\mathrm{PT} / \mathrm{d}-15)]
\end{gathered}
$$

où :

$\mathrm{PN}$ : nombre de positifs à la visite $\mathrm{n}$ et non traités à $\mathrm{n}-1$

$\mathrm{PT}$ : nombre de positifs à la visite $\mathrm{n}$ et traités à $\mathrm{n}-1$

$\mathrm{N}$ : total des animaux non traités à $\mathrm{n}-1$

$\mathrm{T}$ : total des animaux traités à $\mathrm{n}-1$

$\mathrm{d}:$ nombre de jours entre les deux visites

$\mathrm{M}$ : population totale
Malgré l'action trypanopréventive limitée de l'acéturate de diminazène, l'apparition de phénomènes de résistance à ce principe actif relativise la confiance que l'on peut lui accorder. L'estimation de ces résistances dans la zone cotonnière du Burkina Faso est en cours (25).

\section{Suivi du parcours des troupeaux}

Afin de connaître le parcours journalier des animaux, les troupeaux sentinelles ont été suivis sur le terrain durant trois jours consécutifs, en fin de saison sèche et durant la saison des pluies. Leur position a été relevée tous les quarts d'heure au Gps (Global Positioning System, Garmin $\left.75^{\circledR}\right)$. Ces résultats très précis ont été comparés à ceux d'une étude plus générale portant sur les pratiques d'élevage et les stratégies d'utilisation de l'espace (15).

\section{Suivi entomologique}

Les glossines ripicoles ont des exigences écologiques étroites. Elles restent concentrées dans les formations forestières riveraines, notamment durant la saison sèche. Cependant, lors de la période humide, elles ont une certaine aptitude à se disperser dans les savanes avoisinantes à la recherche d'hôtes nourriciers $(10,29)$. Afin d'estimer l'ampleur de cette dispersion, des pièges biconiques et monoconiques du type Vavoua ont été disposés alternativement tous les $100 \mathrm{~m}$ le long de transects allant du point d'abreuvement le plus proche jusqu'au village $(5,6,20)$. La position de chacun de ces pièges a été relevée par Gps, selon une procédure assurant une précision de $20 \mathrm{~m}$ (14). Le dispositif de piégeage a été laissé en place trois jours consécutifs, toutes les deux semaines durant la saison des pluies (de début juillet à début septembre) et les récoltes d'insectes ont été effectuées tous les soirs.

\section{RESU LTATS}

\section{Le site de Nakaka : un contact hôtes-vecteurs permanent}

\section{Des incidences élevées toute l'année}

La figure 2 présente les incidences mensuelles observées dans le troupeau de Nakaka durant les deux années de suivi. Les incidences mensuelles de l'infection à T.vivax se situent pratiquement toute l'année entre 5 et 10 p. 100 , avec des pics jusqu'à 20 p. 100 en saison sèche chaude (mars - avril). Celles de T. congolense sont centrées sur la fin de saison sèche chaude et la saison des pluies (avril à septembre), période durant laquelle les densités de glossines augmentent. La transmission de T. brucei s.l. semble plus accidentelle. Les animaux sont donc en permanence soumis à une forte pression parasitaire.

\section{Des parcours de vaste amplitude}

Les parcours des animaux en fin de saison sèche (juillet) et durant la saison des pluies (septembre) sont représentés sur la figure 3. Chez les Peuls, les animaux sont généralement confiés à un jeune bouvier qui les conduit sur les pâturages et aux points d'abreuvement. Chaque soir, le troupeau rentre au campement.

Au mois de juillet, période de la préparation des cultures, les troupeaux doivent éviter les aires cultivées. Bien qu'il commence à pleuvoir à cette époque, le parcours indiqué ici est représentatif de ceux qui sont utilisés durant la période de sécheresse (15). Les principales contraintes qui guident les déplacements sont la recherche de pâturage et, surtout, des points d'abreuvement (18, 23). Les animaux sont abreuvés une ou deux fois dans la journée aux points d'eau pérennes du réseau hydrographique. 

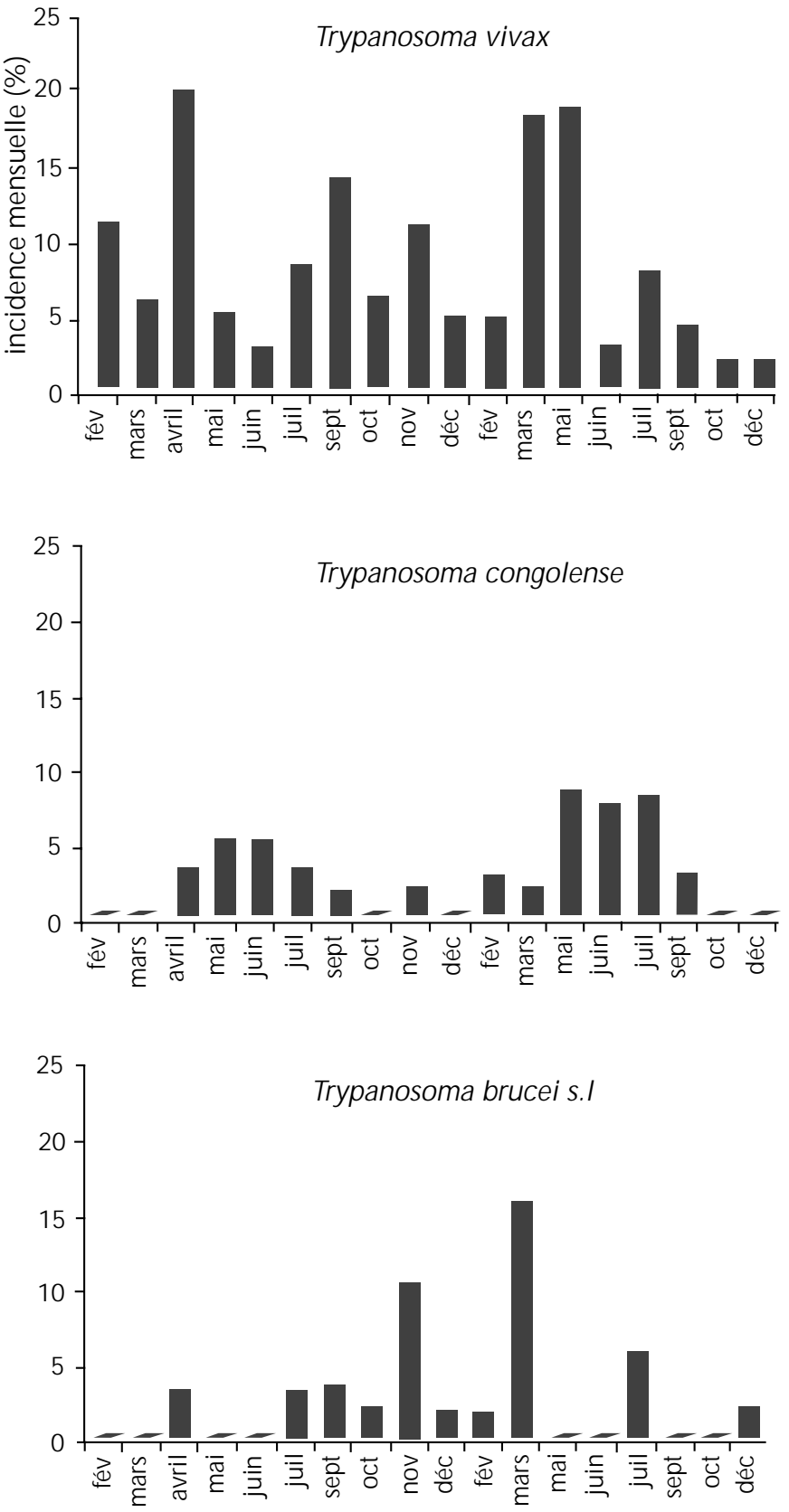

Figure 2 : incidences trypanosomiennes mensuelles (Trypanosoma vivax, T. congolense, T. brucei s.l.) dans le troupeau du village de Nakaka.

$\mathrm{Au}$ mois de septembre, le problème de l'abreuvement est moins crucial car les pluies ont créé de nombreuses mares temporaires. L'espace cultivé se libère après les premières récoltes et les animaux pénètrent dans les champs pour profiter des résidus de cultures. Les parcours sont plus restreints et centrés autour du campement.

\section{La dispersion des glossines}

La dispersion des mouches dans les savanes proches du réseau hydrographique est maximale durant la saison des pluies. Lors du suivi entomologique, $G$. tachinoides a été capturée régulièrement à différents niveaux du transect de pièges, jusque dans le village de Nakaka, pourtant distant de $2 \mathrm{~km}$ (figure 3). Les densités étaient cependant plus élevées dans les pièges proches de la galerie fores- tière. G. palpalis gambiensis a également pu être mise en évidence dans le village et sur le transect, mais à deux occasions seulement. Cette dispersion des mouches est favorisée par la situation du village, localisé le long d'une dépression humide où la végétation arborée est dense. Ces résultats des captures confirment l'aptitude des deux espèces de glossines à sortir de leur biotope de saison sèche et à assurer en période humide une transmission sur le site même du village.

Ainsi, à Nakaka, la transmission des trypanosomes en saison sèche est assurée par un contact étroit et régulier entre les bovins et les glossines aux points d'abreuvement dans la galerie. En saison des pluies, les bovins ne fréquentent plus les points d'eau du réseau hydrographique, mais les mouches se dispersent dans les savanes et peuvent infecter les animaux jusque dans le village.

\section{Le site de Péfrou : des situations variables selon la localisation du campement}

Les résultats parasitologiques obtenus à Péfrou indiquent deux situations très différentes selon la proximité ou l'éloignement entre le campement du propriétaire des bovins et le réseau hydrographique.

\section{Les campements situés à proximité du cours d'eau}

Les courbes d'incidence obtenues chez les animaux localisés au voisinage de la Tolé (moins de $500 \mathrm{~m}$ ) sont reportées dans la figure 4 (9 éleveurs, 33 animaux). Elles indiquent une pression de la transmission parasitaire régulière tout au long de l'année.

Les parcours journaliers des animaux sont de petite amplitude (figure 5). Confiés à un enfant de la famille, les bovins restent en périphérie de la concession, même durant la période de culture. Après les récoltes, ils sont menés sur les parcelles pour consommer les résidus de culture et assurer la fertilisation organique du sol. Dans ces campements situés à proximité du cours d'eau, les animaux sont abreuvés aux puisards creusés dans le lit de la rivière. Le contact avec les glossines est donc fréquent, comme en attestent les captures régulières de $G$. tachinoides à ces endroits.

\section{Les campements situés à l'écart du cours d'eau}

Dans le cheptel des campements situés à plus de $2 \mathrm{~km}$ du réseau hydrographique (11 éleveurs, 41 animaux), les résultats parasitologiques obtenus sont très différents (figure 6). La transmission est exceptionnelle, et les quelques infections détectées surviennent durant les périodes humides.

Ces animaux ne fréquentent pas les cours d'eau. Les effectifs réduits des troupeaux permettent au propriétaire de les abreuver avec l'eau du puits familial situé dans le campement. Ils restent ainsi éloignés des biotopes des glossines (figure 5). Par ailleurs, celles-ci ne se dispersent pas, ou peu, dans les savanes densément occupées par les cultures. Lors du suivi entomologique, seuls deux spécimens ont été capturés le long du transect au cours de la saison des pluies. Ces deux captures correspondaient à des pièges posés dans une zone de végétation dense, dans un bas-fond relié à l'un des affluents du cours d'eau (figure 5).

Ainsi, à Péfrou, les animaux issus des campements proches de la Tolé sont soumis à une forte pression glossinienne qui assure une transmission toute l'année. A l'inverse, les troupeaux des campements éloignés du cours d'eau ne fréquentent pas les biotopes à glossines. De plus celles-ci se dispersent peu dans un espace essentiellement agricole qui leur est très défavorable. Ces animaux ne s'infectent donc pas ou peu. 
Densités de Glossina tachinoides

aucune glossine
de 1 à 3 glossines
de 3 à 6 glossines
de 6 à 30 glossines
$\ldots \ldots$ parcours de fin de saison sèche

$\square$, réseau hydrographique
savane arborée dense
parcelles cultivées

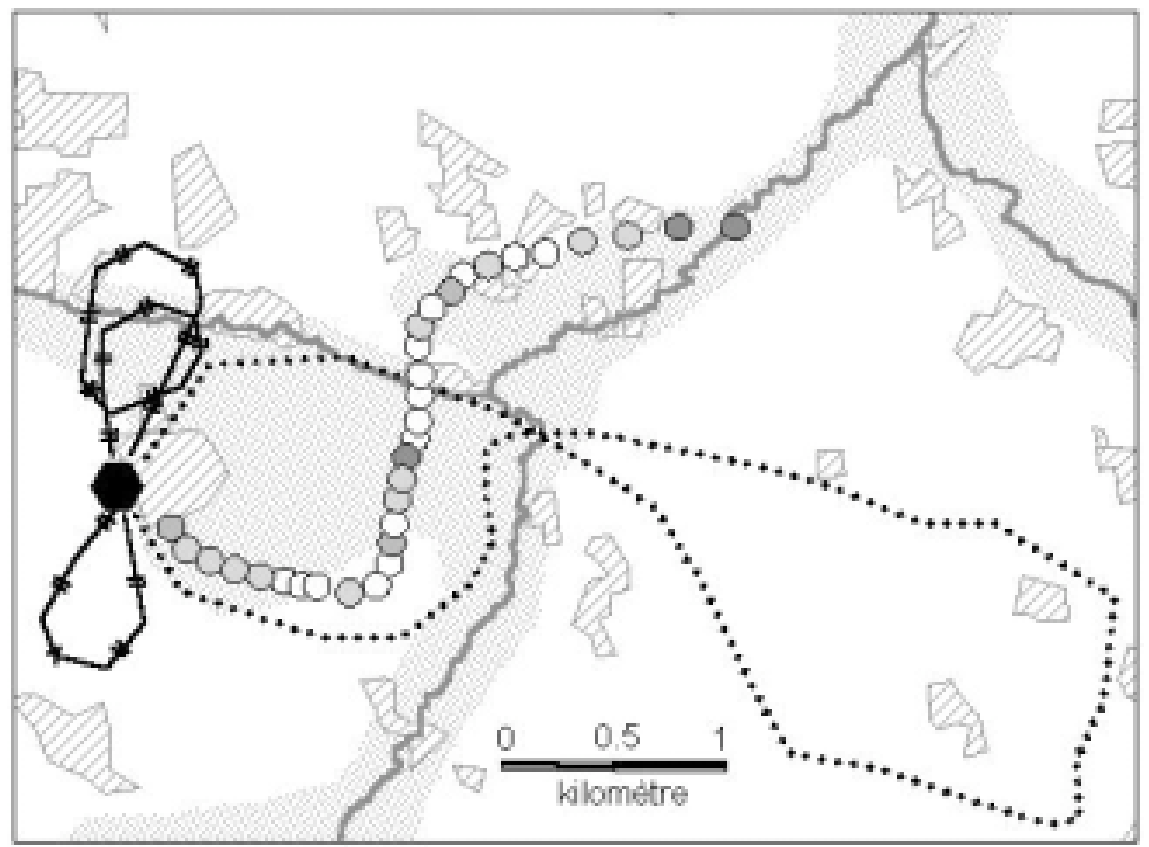

Figure 3 : parcours des animaux du village de Nakaka en fin de saison sèche, en saison des pluies et résultats entomologiques le long du transect.
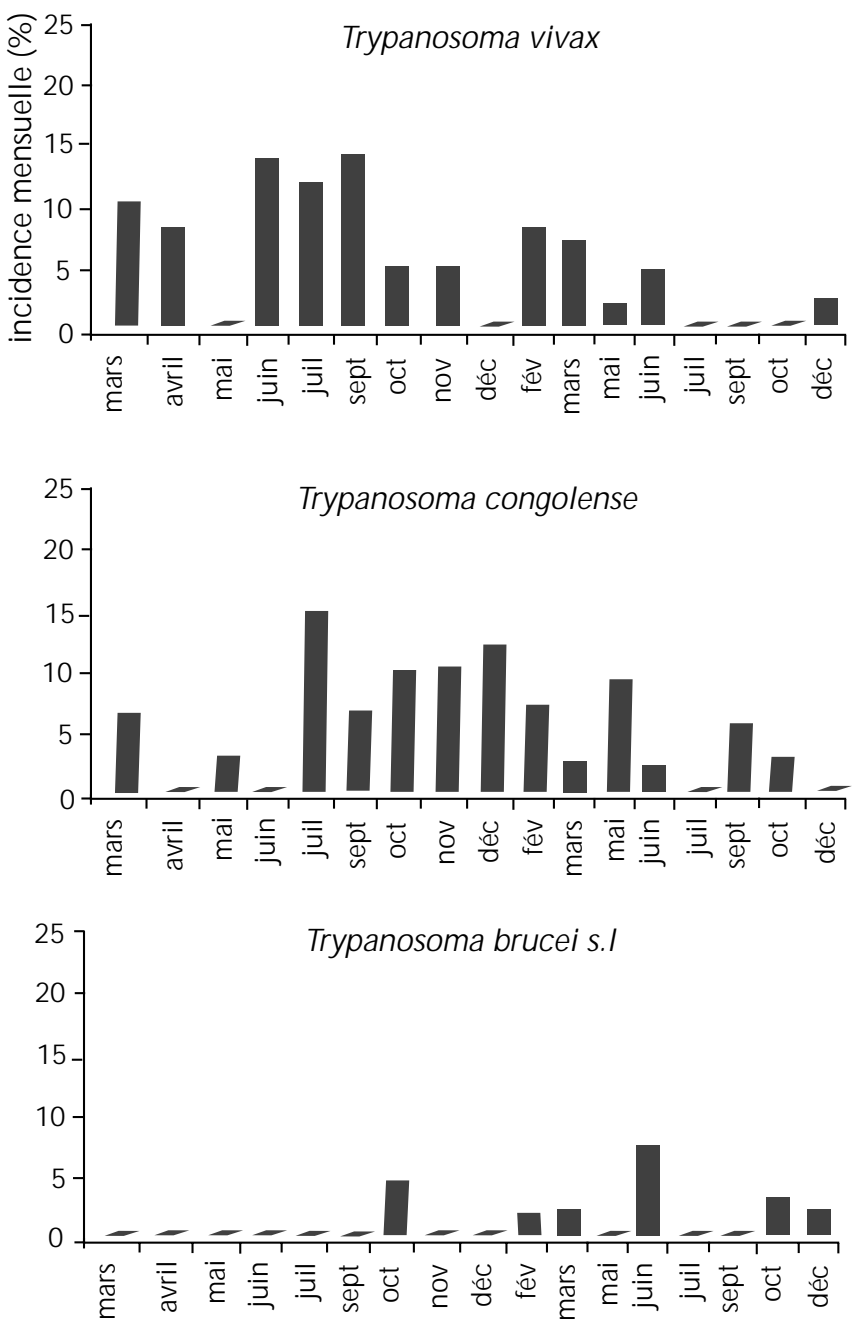

Figure 4 : incidences trypanosomiennes mensuelles (Trypanosoma vivax, T. congolense, T. brucei s. I.) chez les animaux du village de Péfrou issus de campements proches de la rivière.

\section{DISCUSSION}

Dans la littérature, le risque trypanosomien est classiquement relié à l'importance numérique des vecteurs $(7,17,21,27,31)$. Cette relation entre les densités de glossines et la pression de transmission est bien admise avec les mouches des savanes - qui sont largement distribuées et ont des capacités de dispersion saisonnière rapides et amples. De ce fait, leur répartition spatiale est souvent considérée comme homogène. Quelques études ont cependant montré qu'à petite échelle, même avec ces espèces savanicoles, la transmission parasitaire pouvait être hétérogène dans l'espace (30). On a ainsi observé que, dans une savane colonisée par G. morsitans submorsitans, la pression glossinienne peut varier selon un rapport de 1 à 10 en fonction des troupeaux et de leurs lieux de pâture (36).

Dans le cas des glossines ripicoles, les zones de contact entre les vecteurs et les bovins sont encore plus circonscrites. En saison sèche, la répartition des glossines est essentiellement limitée aux formations arborées du réseau hydrographique. Or, la fréquentation des points d'abreuvement situés dans les galeries forestières est une obligation pour les propriétaires de grands troupeaux, qui ne peuvent pourvoir aux besoins de leurs animaux par l'exhaure manuelle. Les agriculteurs propriétaires de petits troupeaux ont, quant à eux, la possibilité de tirer l'eau des puits ou des forages et peuvent éviter les sites dangereux de la rivière. Mais, peut-être en raison d'une mauvaise connaissance de la maladie, ceux d'entre eux installés juste à proximité du réseau hydrographique y abreuvent régulièrement leurs animaux. Cette pratique quotidienne est d'autant plus dangereuse qu'elle permet la mise en place d'un système local de transmission parasitaire entre une population de vecteurs - pas toujours très abondante mais très infectée et infectante - et un troupeau quotidiennement agressé, à la fois source et victime des parasites $(9,22)$. La connaissance des pratiques pastorales et des systèmes d'élevage a donc une très grande importance dans l'évaluation du risque de transmission parasitaire (11).

Durant la saison humide, les glossines peuvent sortir de leurs biotopes traditionnels et se disperser dans les savanes avoisinantes. 


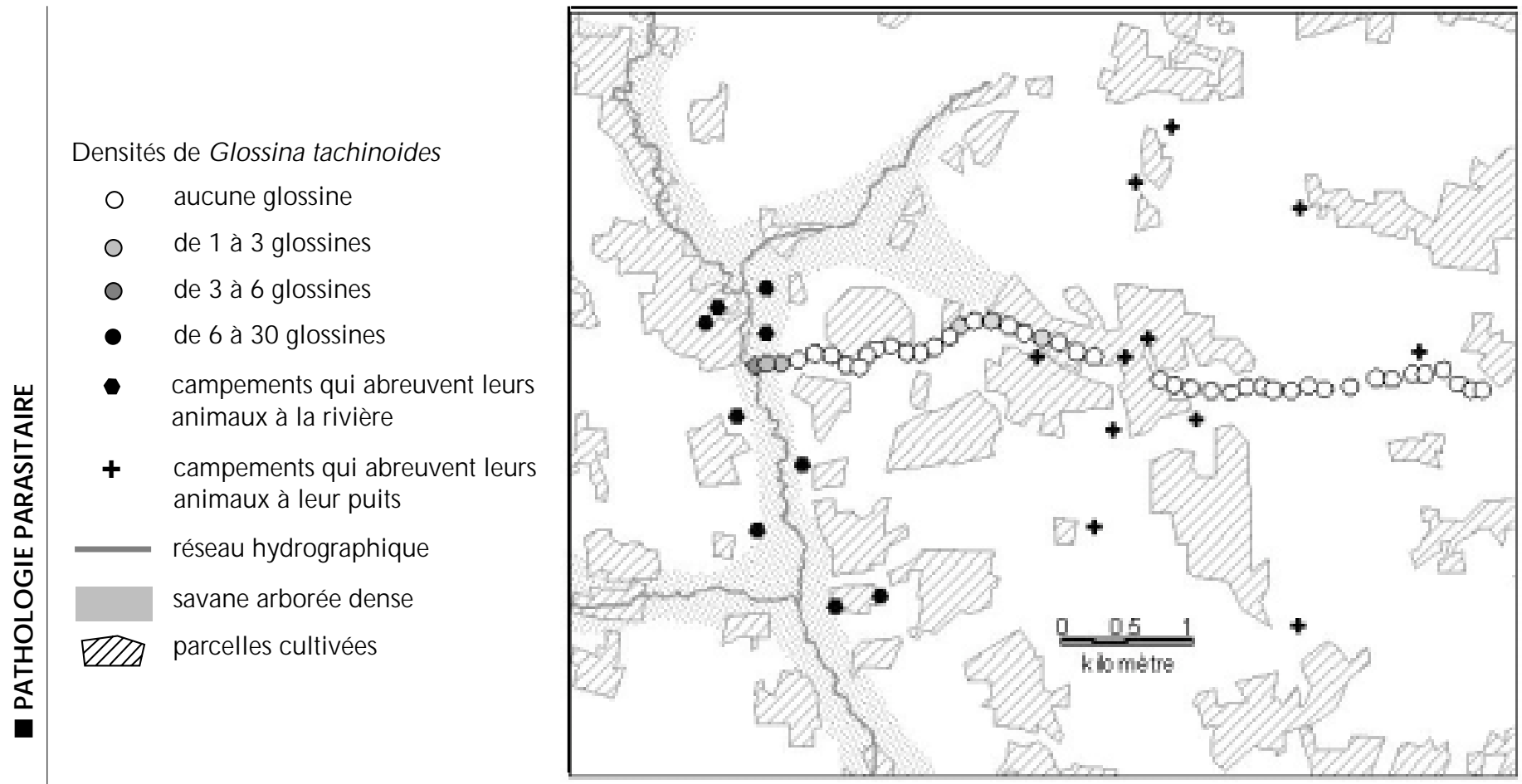

Figure 5 : localisation des campements de Péfrou et résultats entomologiques le long du transect.

Les sites de transmission sont alors déplacés parfois jusque dans les villages eux-mêmes. Cependant cette dispersion des vecteurs dépend des caractéristiques écologiques du milieu savanicole rencontré. Elle est très nette dans le cas de Nakaka, village situé dans une zone pastorale entourée de savane densément arborée, où les glossines (et particulièrement $G$. tachinoides) sont rencontrées régulièrement tout au long du transect. Dans le village, les vecteurs colonisent des gîtes péridomestiques $(1,4,16,19)$ et sont à l'origine de systèmes épidémiologiques temporaires. En revanche, dans les milieux fortement anthropisés la dispersion est très réduite, le défrichement et les cultures créant un milieu défavorable aux glossines. Ces paysages agricoles constituent en quelque sorte une barrière de protection pour les campements à l'écart des biotopes naturels des mouches tsé-tsé.

Lors des séances régulières de piégeage, plusieurs espèces d'insectes hématophages potentiellement vecteurs mécaniques des trypanosomoses animales ont été capturées le long des transects, aux points d'eau situés en savane (Haematopota occidentalis, H. lacessans, Atylotus albipalpus, A. agrestis, Chrysops distinctipennis, Tabanus taeniola, T. par, T. gratus, T. biguttatus, T. ruficrus, Ancala necropina) et autour des parcs de nuit (Stomoxys calcitrans, S. niger, Hippobosca sp.). L'importance de la transmission mécanique est difficile à évaluer dans les zones infectées de glossines, mais ce type de transmission pourrait prendre le relais de la transmission cyclique et amplifier les incidences en saison des pluies, période d'activité de ces insectes (11). Cela suppose néanmoins que certains animaux du troupeau soient initialement infectés. La transmission mécanique pourrait donc avoir un impact épidémiologique à Nakaka, mais ne permet pas d'expliquer les quelques infections relevées dans les campements de Péfrou éloignés de la rivière. D'ailleurs, si la transmission mécanique est efficace pour certains trypanosomes tels que $T$. vivax, elle se réalise plus difficilement avec $T$. congolense et $T$. brucei s. 1. (33), dont les incidences restent en conséquence de bons indi- cateurs de l'interface entre les hôtes vertébrés et les glossines. Les quelques infections observées à Péfrou restent donc difficiles à expliquer, sinon par une fréquentation exceptionnelle des points d'abreuvement du réseau ou la dispersion très occasionnelle des glossines.

\section{CONCLUSION}

L'ensemble de ces résultats permet d'imaginer quelques recommandations à proposer aux éleveurs ou aux agents de santé sur le terrain. La principale serait sans doute de tenir compte de la diversité des systèmes existants et des risques associés. Une bonne connaissance du terrain, l'appréciation du milieu écologique et des pratiques pastorales permettent de reconnaître, à l'échelle d'un terroir, les situations les plus dangereuses et de proposer les interventions adaptées. Finalement, la compréhension du système de transmission impose de s'intéresser à des thèmes qui dépassent le contexte bio-écologique classiquement décrit. La gestion spatiale des troupeaux, la transformation des paysages, donc la dynamique des interfaces hôtes/vecteurs/parasites, reposent en partie sur les contraintes et les motivations des propriétaires. Celles-ci peuvent être à la fois sociales, culturelles, ethniques ou économiques. Considérer le milieu, au sens large, comme un révélateur du fonctionnement du système pathogène semble particulièrement adapté à l'épidémiologie complexe des trypanosomoses transmises par les glossines ripicoles.

\section{Remerciements}

Les auteurs remercient le directeur général du Cirdes S.M. Toure, pour son appui au déroulement de ce travail. L'étude a été financée par le Cirad (Action thématique programmée Santé, environnement) et le Cnrs (Programme Environnement, vies et sociétés). 

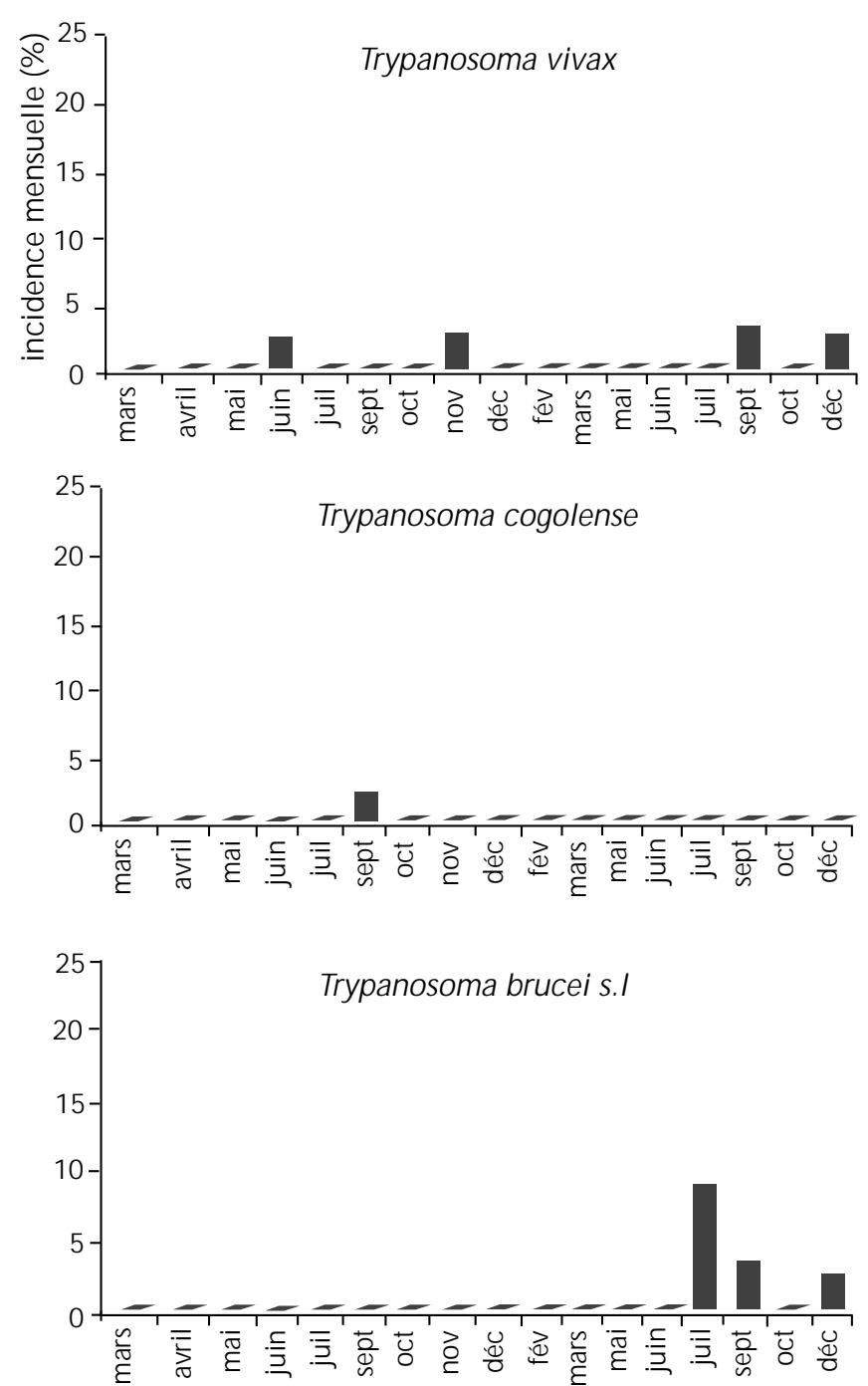

Figure 6 : incidences trypanosomiennes mensuelles (Trypanosoma vivax, T. congolense, T. brucei s.l.) chez les animaux du village de Péfrou issus de campements éloignés de la rivière.

\section{BIBLIO GRAPHIE}

1. BALDRY D.A.T., 1970. Observations on the peridomestic breeding behaviour and resting sites of Glossina tachinoides W est. near N sukka, East Central State, Nigeria. Bull. Entomol. Res., 59: 585-593.

2. BEN OIT M., 1977. Introduction à la géographie des aires pastorales soudaniennes de Haute-Volta. Paris, France, O rstom, 95 p.

3. CAMUS E., 1983. Diagnostic de la trypanosomose bovine sur le terrain par la méthode de centrifugation hématocrite. Rev. sci. tech. $0 \mathrm{ff}$. int. Epiz., 2 : 751-769.

4. CHALLIER A., 1980. Ecology and epidemiological importance of Glossina palpalis in the Ivory Coast forest zone. Insect Sci. Appl., 1: 77-83.

5. CHALLIER A., EYRAUD M., LAFAYE A., LAVEISSIERE C., 1977. Amélioration du rendement du piège biconique pour glossines (Diptera, Glossinidae) par l'emploi d'un cône inférieur bleu. Cah. O rstom Sér. Ent. méd. Parasitol., 15 : 283-286.

6. CHALLIER A., LAVEISSIERE C., 1973. Un nouveau piège pour la capture des glossines (Glossina: Diptera - Muscidae), description et essais sur le terrain. Cah. O rstom Sér. Ent. méd. Parasitol., 11 : 251-262.

7. CLAXTON J.R., LEPERRE P., RAWLINGS W.F., SNOW W.F., DW IN GER R.H., 1992. Trypanosomiasis in cattle in Gambia : incidence, prevalence and tsetse challenge. Acta trop., 50: 219-225.
8. COMBES C., 1995. Interactions durables, écologie et évolution du parasitisme. Paris, France, Masson, 524 p.

9. CUISAN CE D., DE LA RO CQUE S., 1998. Tsé-tsé et trypanosomoses: du système pathogène à l'évaluation du risque. Montpellier, France, Cirad-emvt/Cnrs, $90 \mathrm{p}$.

10. CUISANCE D., FEVRIER J., DEJARDIN J., FILLEDIER J., 1985. Dispersion linéaire de Glossina palpalis gambiensis et $\mathrm{G}$. tachinoides dans une galerie forestière en zone soudano-guinéenne (Burkina Faso). Revue Elev. Med. vét. Pays trop., 47 : 69-75.

11. D'AMICO F., 1993. Rôle de Glossina fuscipes fuscipes Newstead, 1910, dans la transmission des trypanosomoses bovines en Afrique centrale. Cas de la zone d'élevage d'Ouro-Djafoun (République centrafricaine). Thèse Doct., Université Montpellier II, Montpellier, France, $160 \mathrm{p}$.

12. DE LA ROCQUE S., 1997. Identification des facteurs discriminants de la présence de glossines dans une zone agro-pastorale du Burkina Faso. Intérêt pour l'évaluation du risque trypanosomien. Thèse Doct. Université M ontpellier II, M ontpellier, France, 162 p.

13. DESQ UESNES M., DE LA ROCQUE S., 1995. Comparaison de la sensibilité du test de $W 00$ et d'un test de détection des antigènes de Trypanosoma vivax chez des moutons expérimentalement infectés avec une souche guyanaise du parasite. Rev. Elev. Méd. vét. Pays trop., 48 : 247-253

14. FREYCON V., FAUVET N., 1998. Les Gps (Global Positioning System) : de l'acquisition des relevés à leur intégration dans un Sig. Montpellier, France, Cirad. 70 p.

15. INERA, 1998. Etude des systèmes d'élevage dans la Zap de Sidéradougou : pratiques d'élevage intégrées aux exploitations agricoles et stratégies d'utilisation de l'espace. Bobo-Dioulasso, Burkina Faso, Inera/Cirdes/Cirad, 28 p.

16. KUZOE F.A.S., BALDRY D.A.T., VAN DER VLOEDT A., CULLENS J.R., 1985. O bservation of an apparent population extension of Glossina tachinoides W estw. in Southern Ivory Coast. Insect Sci. Appl., 6: 55-58.

17. LAMBRECHT F.L., 1980. Ecological and physiological factors in the cyclic transmission of African Trypanosomiasis. Insect Sci. Appl., 1: 47-54.

18. LANDAIS E., 1983. Analyse des systèmes d'élevage sédentaires du nord de la Côte d'Ivoire. Maisons-Alfort, France, Cirad-iemvt, 759 p.

19. LAVEISSIERE C., COURET D., HERVOUET J.P., 1986. Localisation et fréquence du contact homme/glossines en secteur forestier de Côte d'Ivoire. 1. Recherche des points épidémiologiquement dangereux dans I'environnement végétal. Cah. O rstom Sér. Ent. méd. Parasitol., 24 : 21-35.

20. LAVEISSIERE C., GREBAUT P., 1983. Recherches sur les pièges à glossines (Diptera, Glossinidae). Mise au point d'un modèle économique : le piège «Vavoua ». Trop. Med. Parasitol., 41 : 185-192.

21. LEAK S.G.A., MULATU W., AUTHIE E., D'IETEREN G.D.M., PEREG RINE A.S., ROW LANDS G.J., TRAIL J.C.M., 1993. Epidemiology of bovine trypanosomiasis in the Guide valley, Southwest Ethiopia. 1. Tsetse challenge and its relationship to trypanosome prevalence in cattle. Acta trop., 53: 121-134.

22. LEFRANÇOIS T., SOLANO P., DE LA ROCQUE S., BENGALY Z., REIFENBERG J.M., KABORE I., CUISANCE D., 1998. N ew epidemiological data on animal trypanosomosis by molecular analysis in the pastoral zone of Sideradougou, Burkina Faso. M ol. Ecol., 7: 897-904. 23. LHOSTE P., DOLLE V., ROUSSEAU J., SOLTNER D., 1993. Manuel de zootechnie des régions chaudes : les systèmes d'élevage. Paris, France, ministère de la Coopération, 288 p. (Coll. Manuels et précis d'élevage)

24. MCLENNAN K.J.R., 1971. The aparasitemic interval following diminazene aceturate therapy of a relapsing strain of $T$. vivax infecting cattle. Trop. Anim. Health Prod., 3: 208-212.

25. M CDERM OTT J.J., WOITAG T., BAUER B., SIDIBE I., O UEDRAOGO D., KAMUANGA J.M.B., CLAUSEN P.H., EISLER M., PEREGRINE A.S., ZESSIN K.H., MEHLITZ D., 1999. Le risque d'infection trypanosomienne, la pression glossinaire et la résistance aux trypanocides dans la province du Kénédougou, Burkina Faso. In : $25^{\mathrm{e}}$ réunion Csirlt, 27 sept.-1 oct. 1999, Mombasa, Kenya, O ua/Cstr.

26. MICHEL J.F., MICHEL V., DE LA ROCQUE S., TOURE I., RICHARD D., 1999. Modélisation de l'occupation de l'espace par les bovins. Applications à l'épidémiologie des trypanosomoses animales. Rev. Elev. Méd. vét. Pays trop., 52 : 25-33. 
27. MILLIGAN P.M.J., BAKER R.D., 1988. A model of tsetse-transmitted animal trypanosomiasis. Parasitol., 96: 211-239.

28. MURRAY M., MURRAY P.K., MCINTYRE W.I.M., 1977. An improved parasitological technique for the diagnosis of African trypanosomiasis. Trans. R. Soc. Trop. Med. Hyg., 71: 325-326.

29. POLITZAR H., CUISANCE D., CLAIR M., TAZE Y., SELLIN E., BOURDOISEAU G., 1980. Etude préliminaire de la longévité et de la dispersion des mâles irradiés de Glossina palpalis gambiensis dans leur biotope naturel (Haute-Volta). Rev. Elev. Méd. vét. Pays trop., 33 : 45-50.

30. RAW LINGS P., DWINGER R.H., SNOW W.F., 1991. An analysis of survey measurement of tsetse challenge to trypanotolerant cattle in relation to aspect of analytical models of trypanosomiasis. Parasitol., 102: 371-377.

31. ROGERS D.J., 1985. Trypanosomiasis « risk » or « challenge »: a review. Acta trop., 42: 5-32.

32. SOLANO P., MICHEL J.F., LEFRANÇOIS T., DE LA ROCQUE S. SIDIBE I., ZOUNGRANA A., CUISANCE D., 1999. Polymerase chain reaction as a diagnosis tool for detecting trypanosomes in naturally infected cattle in Burkina Faso. Vet. Parasitol., 86: 95-103.
33. TRO NCY P.M., ITARD J., MOREL P.C., 1981. Précis de parasitologie tropicale. Paris, France, ministère de la Coopération, $717 \mathrm{p}$.

34. UILENBERG G., 1998. A field guide for the diagnosis, treatment and prevention of African animal trypanosomosis. Rome, Italie, Fao, 158 p.

35. VERY P., BOCQUENTIN R., DUVALLET G., 1990. Sensibilité de la double microcentrifugation pour la recherche des trypanosomes. Rev. Elev. Méd. vét. Pays trop., 43 : 325-329.

36. WACHER T.J., MILLIGAN P.J.M., RAW LINGS P., SNOW W.F., 1994. Tsetse-trypanosomiasis challenge to village N'Dama cattle in the Gambia : field assessment of spatial and temporal patterns of tsetse-cattle contact and the risk of trypanosomiasis infection. Parasitol., 109: 149162.

37. WHITELAW D.D., GARDINER P.R., MURRAY M., 1988. Extravascular foci of Trypanosoma vivax in goats: the central nervous system and aqueous humor of the eyes as potential source of relapse infection after chemotherapy. Parasitol., 97: 51-61.

Reçu le 10.12.99, accepté le 17.04.00

\section{Summary}

De La Rocque S., Bengaly Z., Michel J.F., Solano P., Sidibé I., Cuisance D. Evaluation of spatial and temporal contacts between tsetse flies and cattle, a key factor for trypanosomiasis transmission in West Africa

A global study was carried out in the agropastoral zone of Sideradougou (Burkina Faso) on risk assessment of trypanosomiasis transmission. Sentinel herds from two different farming systems were sampled and followed up during two years. The monthly incidences of infections were measured and interpreted according to the movements of herds, their practices for watering and their contacts with the riparian tsetse flies (Glossina tachinoides and G. palpalis gambiensis). In Nakaka, a Fulani breeders' village 4 km away from the river, the parasites are transmitted during the dry season at the point of permanent watering places inside the forest gallery. In rainy season, the tsetse flies disperse through the savannah and infect cattle even in the village. In Pefrou, a group of farmers' settlements, herds are made up mainly of draught cattle. Animals from settlements located near the hydrographic network drink in the river and are regularly infected all the year long. The incidences are higher during the rainy season and the beginning of the dry season when tsetse flies are the most abundant. On the other and, herds from the settlements far away $(3 \mathrm{~km})$ from the hydrographic network are watered from wells and do not frequent the habitats of vectors. In such an agricultural landscape, tsetse flies do not disperse even in the rainy season. Incidences in these herds are almost nil. These results prove the importance of space and temporal contacts between hosts and vectors in the epidemiology of trypanosomiasis in W est Africa.

Key words: Cattle - Glossina - Trypanosomiasis Epidemiology - Environment - Agriculture - Animal husbandry methods - Burkina Faso.

\section{Resumen}

De La Rocque S., Bengaly Z., Michel J.F., Solano P., Sidibé I., Cuisance D. Importancia de las interfaces espaciales y temporales entre los bovinos y las glossinas en la transmisión de la tripanosomiasis animal en Africa del 0 este

Durante un estudio global sobre el riesgo del tripanosoma, llevado a cabo en la zona agropastoril de Sidéradougou (Burkina Faso), se siguieron durante dos años hatos centinelas originarios de dos sistemas de crianza diferentes. La incidencia tripanosómica mensual se midió e interpretó en función del trayecto de los hatos, de las prácticas para abrevar y del contacto con las glossinas ripícolas (Glossina tachinoides y G. palpalis gambiensis). En Nakaka, pueblo de criados Peul, la transmisión durante la estación seca es segura en los puntos de abrevadero perennes en la galería forestal. Durante la estación lluviosa, las glossinas ripícolas se dispersan en las sabanas e infectan los animales, incluso hasta en el pueblo. En Pefrou, conjunto de campamentos de agricultores Bobo, los hatos están compuestos esencialmente de bueyes de tracción. Los animales originarios de los campamentos localizados a proximidad de los cursos de agua abrevan en los puntos de agua de las formaciones ribereñas y se infectan regularmente todo el año. Los incidentes son más frecuentes durante el invierno y al inicio de la estación seca, periodo en el cual las glossinas son más numerosas. Por otro lado, los hatos de los campamentos alejados de la red hidrográfica $(3 \mathrm{~km})$ abrevan en pozos y no frecuentan los biotopos de las glossinas. En este panorama, esencialmente agrícola, las glossinas no se dispersan, ni siquiera durante la estación húmeda. Los incidentes en los hatos son casi inexistentes. Los resultados muestran la importancia de las interfaces espaciales y temporales entre los bovinos y las glossinas en la epidemiología de las tripanosomiasis en Africa del 0 este.

Palabras clave: Ganado bovino - Glossina - Tripanosomiasis Epidemiología - Medio ambiente - Agricultura - Métodos de crianza - Burkina Faso. 